\title{
Complex Exceptional Orthogonal Polynomials and Quasi-invariance
}

\author{
WILLIAM A. HAESE-HILL ${ }^{1}$, MARTIN A. HALLNÄS ${ }^{1}$ and \\ ALEXANDER P. VESELOV ${ }^{1,2}$ \\ ${ }^{1}$ Department of Mathematical Sciences, Loughborough University, Loughborough \\ LE113TU, UK.e-mail: M.A.Hallnas@lboro.ac.uk \\ ${ }^{2}$ Moscow State University, Moscow, Russia.
}

Received: 12 October 2015 / Revised: 30 December 2015 / Accepted: 1 February 2016 Published online: 14 March 2016 - (C) The Author(s) 2016. This article is published with open access at Springerlink.com

Abstract. Consider the Wronskians of the classical Hermite polynomials

$$
H_{\lambda, l}(x):=\operatorname{Wr}\left(H_{l}(x), H_{k_{1}}(x), \ldots, H_{k_{n}}(x)\right), \quad l \in \mathbb{Z}_{\geq 0} \backslash\left\{k_{1}, \ldots, k_{n}\right\},
$$

where $k_{i}=\lambda_{i}+n-i, i=1, \ldots, n$ and $\lambda=\left(\lambda_{1}, \ldots, \lambda_{n}\right)$ is a partition. Gómez-Ullate et al. showed that for a special class of partitions the corresponding polynomials are orthogonal and dense among all polynomials with respect to a certain inner product, but in contrast to the usual case have some degrees missing (so-called exceptional orthogonal polynomials). We generalise their results to all partitions by considering complex contours of integration and non-positive Hermitian products. The corresponding polynomials are orthogonal and dense in a finite-codimensional subspace of $\mathbb{C}[x]$ satisfying certain quasiinvariance conditions. A Laurent version of exceptional orthogonal polynomials, related to monodromy-free trigonometric Schrödinger operators, is also presented.

Mathematics Subject Classification. 33C47 (81Q05).

Keywords. exceptional orthogonal polynomials, quasi-invariance, trivial monodromy, Darboux transformations.

\section{Introduction}

Consider polynomials $p_{n}(x) \in \mathbb{R}[x]$ of degrees $n=0,1, \ldots$, satisfying the orthogonality relation

$$
\left(p_{m}, p_{n}\right)=\delta_{m n} g_{n},
$$

where the inner product of polynomials is defined by a real integral

$$
(p, q):=\int_{a}^{b} p(x) q(x) w(x) \mathrm{d} x
$$


for some positive weight function $w$. Suppose that there exists a second-order differential operator

$$
T=A(x) \frac{\mathrm{d}^{2}}{\mathrm{~d} x^{2}}+B(x) \frac{\mathrm{d}}{\mathrm{d} x}+C(x)
$$

having these polynomials as eigenvectors:

$$
T p_{n}(x)=E_{n} p_{n}(x), \quad n=0,1, \ldots
$$

A classical result due to Bochner [3] says that in that case the sequence of polynomials $p_{n}(x), n \in \mathbb{Z}_{\geq 0}$, must coincide (up to a linear change of $x$ ) with one of the systems of classical orthogonal polynomials of Hermite, Laguerre or Jacobi.

Gómez-Ullate, Kamran and Milson [11] considered the following variation of Bochner's question. Let us assume now that in the previous considerations $n$ belongs to a certain proper subset $S \subset \mathbb{Z}_{\geq 0}$ such that $\mathbb{Z}_{\geq 0} \backslash S$ is finite. To make this non-trivial they added the following density condition: the linear span $U=\left\langle p_{n}: n \in\right.$ $S\rangle$ of the corresponding polynomials must be dense in $\mathbb{R}[x]$ in the sense that if $\left(p, p_{n}\right)=0$ for all $n \in S$ then $p \equiv 0$. In that case the sequence $p_{n}(x), n \in S$ is called a system of exceptional orthogonal polynomials.

The main example of such polynomials are exceptional Hermite polynomials [12] having the Wronskian form

$$
H_{\lambda, l}(x):=\operatorname{Wr}\left(H_{l}(x), H_{k_{1}}(x), \ldots, H_{k_{n}}(x)\right), \quad l \in \mathbb{Z}_{\geq 0} \backslash\left\{k_{1}, \ldots, k_{n}\right\},
$$

where $H_{l}(x)$ are classical Hermite polynomials, $\lambda=\left(\lambda_{1}, \ldots, \lambda_{n}\right)$ is a double partition and

$$
k_{i}=\lambda_{i}+n-i, \quad i=1, \ldots, n .
$$

The double partitions have the very special form

$$
\lambda=\mu^{2}=\left(\mu_{1}, \mu_{1}, \mu_{2}, \mu_{2}, \ldots, \mu_{k}, \mu_{k}\right),
$$

where $\mu=\left(\mu_{1}, \mu_{2}, \ldots, \mu_{k}\right)$ is another partition with $n=2 k$ (see [8]). According to Krein and Adler [1] this guarantees that the corresponding Wronskian

$$
W_{\lambda}(x)=\operatorname{Wr}\left(H_{k_{1}}(x), \ldots, H_{k_{n}}(x)\right)
$$

has no zeroes on the real line and thus determines a non-singular weight function

$$
w(x)=W_{\lambda}^{-2}(x) e^{-x^{2}} .
$$

The geometry of the complex zeroes of the corresponding Wronskians is quite interesting and was studied by Felder et al. in [8].

One of the goals of our paper is to find a proper interpretation of the exceptional Hermite polynomials (2) for all partitions $\lambda$. As we will see, this will 
naturally lead us to the notion of quasi-invariance, which appeared in the theory of monodromy-free Schrödinger operators, going back to Picard and Darboux and more recently revisited by Duistermaat and Grünbaum [7]. In certain classes such operators were explicitly described in terms of Wronskians in $[4,7,10,15]$. Grinevich and Novikov studied the spectral properties of these and more general singular finite-gap operators and emphasised the important link with the theory of Pontrjagin spaces (see [14] and references therein). Our paper can be considered as dealing with the implications of all these results for the theory of exceptional orthogonal polynomials.

More precisely, we first complexify the picture by considering the vector space $V=\mathbb{C}[z]$ and replace the inner product (1) by a Hermitian product of the form

$$
\langle p, q\rangle:=\int_{C} p(z) \bar{q}(z) w(z) \mathrm{d} z,
$$

where $\bar{q}(z):=\overline{q(\bar{z})}$ is the Schwarz conjugate of the polynomial $q(z), C \subset \mathbb{C}$ is a contour in the complex domain and $w(z)$ is a complex weight function. The condition that this product is Hermitian implies certain restrictions on the contour $C$ and function $w(z)$ (see Section 2). It also requires certain restrictions on the set of polynomials for which the product is well defined. As it turned out, such polynomials form a subspace $U \subset V$ of finite codimension defined by some quasiinvariance conditions. Similarly to [11] we say that the polynomials $p_{n}(z), n \in S$, form a system of complex exceptional orthogonal polynomials if their linear span is a subspace of $U$ that is dense in $U$ in the sense that $\left\langle p, p_{n}\right\rangle=0$ for all $n \in S$, implies that $p \equiv 0$.

We will show that the Wronskians (2) satisfy these criteria for every partition $\lambda$ and a suitable choice of $C$ with $w$ given by (4). For a double partition $\lambda$ we can take as a contour $C$ the real line with $U=V$ and recover the results by GómezUllate et al. [12].

Note that the corresponding Hermitian form is positive definite only for double partitions, otherwise we always have polynomials with negative norms. The appearance of negative norms for singular potentials was first emphasised by Grinevich and Novikov [14].

We also consider the Laurent version of our approach. Some Laurent versions of orthogonal polynomials are already known in the literature (see e.g. [6] and references therein), but our approach is different since it is not based on the GramSchmidt procedure. Similarly, it does not fit into the theory of orthogonal polynomials on the unit circle initiated by Szegö [18], who considered the case of usual polynomials.

Consider the Laurent polynomials $\Lambda=\mathbb{C}\left[z, z^{-1}\right]$ and the following complex bilinear form on $\Lambda$ :

$$
(P, Q)=\frac{1}{2 \pi i} \oint_{C} P(z) Q(z) \frac{\mathrm{d} z}{z},
$$


where $C=\{z \in \mathbb{C}:|z|=1\}$ is the unit circle. The standard basis $z^{n}, n \in \mathbb{Z}$, satisfies the Laurent orthogonality relation

$$
\left(z^{k}, z^{l}\right)=\delta_{k+l, 0}, \quad k, l \in \mathbb{Z} .
$$

We consider more general forms

$$
(P, Q)=\frac{1}{2 \pi i} \oint_{C_{\mu}} P(z) Q(z) w(z) \frac{\mathrm{d} z}{z},
$$

where $C_{\mu}$ is the circle defined by $|z|=\mu$ and $w(z)=W(z)^{-2}$, with $W(z)$ some Laurent polynomial. For this form to be well defined, we need to assume that $P, Q$ belong to a suitable subspace of quasi-invariants $\mathscr{Q} \subset \Lambda$ of finite codimension.

Let $\mathcal{K}$ be a finite subset of $\mathbb{N}$. Suppose that $P_{n} \in \Lambda, n \in \mathbb{Z}$, satisfy the Laurent orthogonality relation

$$
\left(P_{k}, P_{l}\right)=\delta_{k+l, 0} h_{k}, \quad k, l \in \mathbb{Z},
$$

but $P_{n}$ is proportional to $P_{-n}$ for $n \in \mathcal{K}$, which implies that the corresponding $h_{n}=0$, and thus $P_{n}$ is orthogonal to all $P_{k}, k \in \mathbb{Z}$. If the minimal complex Euclidean extension of the linear span of $P_{n}, n \in \mathbb{Z}$, coincides with the subspace of quasiinvariants $\mathscr{Q}$, then we call them exceptional Laurent orthogonal polynomials. The need to consider such an extension is the novelty of the Laurent case, which is related to the fact that the corresponding form is degenerate on the linear span of $P_{n}, n \in \mathbb{Z}$.

We present an example of such polynomials corresponding to the trigonometric monodromy-free Schrödinger operators [4]. Namely, for any set $\kappa=\left\{k_{1}, \ldots, k_{n}\right\}$ of distinct natural numbers $k_{1}>k_{2}>\cdots>k_{n}>0$ and any choice of complex parameters $a=\left(a_{1}, \ldots, a_{n}\right), a_{k} \in \mathbb{C} \backslash\{0\}$, we define the Laurent polynomials

$$
P_{\kappa, a ; l}(z)=\left|\begin{array}{ccccc}
\Phi_{k_{1}}\left(a_{1} ; z\right) & \Phi_{k_{2}}\left(a_{2} ; z\right) & \cdots & \Phi_{k_{n}}\left(a_{n} ; z\right) & z^{l} \\
D \Phi_{k_{1}}\left(a_{1} ; z\right) & D \Phi_{k_{2}}\left(a_{2} ; z\right) & \cdots & D \Phi_{k_{n}}\left(a_{n} ; z\right) & D z^{l} \\
\vdots & \vdots & \ddots & \vdots & \vdots \\
D^{n} \Phi_{k_{1}}\left(a_{1} ; z\right) & D^{n} \Phi_{k_{2}}\left(a_{2} ; z\right) & \cdots & D^{n} \Phi_{k_{n}}\left(a_{n} ; z\right) & D^{n} z^{l}
\end{array}\right|
$$

where $\Phi_{k}(a ; z)=a z^{k}+a^{-1} z^{-k}, k \in \mathbb{N}$ and $D=z \frac{\mathrm{d}}{\mathrm{d} z}$.

When parameters $a_{k}$ satisfy the condition $\left|a_{k}\right|=1$ for all $k=1, \ldots n$ we introduce a Hermitian form on a certain subspace of quasi-invariant Laurent polynomials $\mathscr{Q}_{\kappa, C}$ and show that the minimal Hermitian extension of the linear span of $P_{\kappa, a ; l}$, $l \in \mathbb{Z}$, coincides with the subspace of quasi-invariants $\mathscr{Q}_{\kappa}$ and is dense in $\mathscr{Q}_{\kappa, C}$.

\section{Complex Exceptional Hermite Polynomials}

In this section, we consider the polynomials $H_{\lambda, l}$, as defined in (2), for general partitions, i.e. we do not require that $\lambda$ is a double partition. We shall refer to 
these polynomials as complex exceptional Hermite polynomial or CEHPs for short. Although these polynomials have real coefficients for the partitions which are not double it is natural to consider them as elements of the complex Hermitian vector space because the contour of integration in (5) is complex.

We begin by recalling how $H_{\lambda, l}$ are obtained by a sequence of Darboux transformations from the classical Hermite polynomial $H_{l}$. The starting point is the classical fact that the functions

$$
\psi_{l}(z)=H_{l}(z) e^{-z^{2} / 2}, \quad l \in \mathbb{Z}_{\geq 0}
$$

have the eigenfunction property

$$
\mathscr{L} \psi_{l} \equiv-\frac{\mathrm{d}^{2} \psi_{l}}{\mathrm{~d} z^{2}}+z^{2} \psi_{l}=(2 l+1) \psi_{l}, \quad z \in \mathbb{C},
$$

and satisfy the boundary conditions

$$
\lim _{\operatorname{Re} z \rightarrow \infty} \psi_{l}(z)=0
$$

(for any fixed value of $\operatorname{Im} z$ ). We will choose the normalisation of Hermite polynomials such that the highest coefficient of $H_{l}(z)$ is $2^{l}$ and all the coefficients are integer:

$$
H_{0}=1, H_{1}=2 z, H_{2}=4 z^{2}-2, H_{3}=8 z^{3}-12 z, H_{4}=16 z^{4}-48 z^{2}+12, \ldots .
$$

As is well known, after $n$ consecutive Darboux transformations at the levels $k_{n}<$ $k_{n-1}<\cdots<k_{1}$, where $k_{i}=\lambda_{i}+n-i, i=1, \ldots, n$, one arrives at the Schrödinger operator

$$
\mathscr{L}_{\lambda}=-\frac{\mathrm{d}^{2}}{\mathrm{~d} z^{2}}-2 \frac{\mathrm{d}^{2}}{\mathrm{~d} z^{2}}\left(\log \operatorname{Wr}\left(\psi_{k_{1}}, \ldots, \psi_{k_{n}}\right)\right)+z^{2},
$$

which satisfies the intertwining relation

$$
\mathscr{D}_{\lambda} \circ \mathscr{L}=\mathscr{L}_{\lambda} \circ \mathscr{D}_{\lambda},
$$

where the intertwining operator $\mathscr{D}_{\lambda}$ acts according to

$$
\mathscr{D}_{\lambda} \psi=\frac{\operatorname{Wr}\left(\psi, \psi_{k_{1}}, \ldots, \psi_{k_{n}}\right)}{\operatorname{Wr}\left(\psi_{k_{1}}, \ldots, \psi_{k_{n}}\right)},
$$

see e.g. $[1,5]$. It follows that the functions

$$
\psi_{\lambda, l}=\frac{\operatorname{Wr}\left(\psi_{l}, \psi_{k_{1}}, \ldots, \psi_{k_{n}}\right)}{\operatorname{Wr}\left(\psi_{k_{1}}, \ldots, \psi_{k_{n}}\right)}, \quad l \notin\left\{k_{1}, \ldots, k_{n}\right\},
$$

have the eigenfunction property

$$
\mathscr{L}_{\lambda} \psi_{\lambda, l}=(2 l+1) \psi_{\lambda, l}
$$


Substituting (9) in (12) and using the general property $\operatorname{Wr}\left(g f_{1}, \ldots, g f_{n}\right)=$ $g^{n} \operatorname{Wr}\left(f_{1}, \ldots, f_{n}\right)$, one finds that

$$
\psi_{\lambda, l}=H_{\lambda, l} \frac{e^{-z^{2} / 2}}{W_{\lambda}},
$$

where $H_{\lambda, l}$ are given by (2).

By a direct computation, it is readily inferred that $H_{\lambda, l}$ is an eigenfunction of the operator

$$
T_{\lambda}=-\frac{\mathrm{d}^{2}}{\mathrm{~d} z^{2}}+2\left(z+\frac{W_{\lambda}^{\prime}}{W_{\lambda}}\right) \frac{\mathrm{d}}{\mathrm{d} z}-\left(\frac{W_{\lambda}^{\prime \prime}}{W_{\lambda}}+2 z \frac{W_{\lambda}^{\prime}}{W_{\lambda}}\right)
$$

with eigenvalue $2(l-n)$.

EXAMPLE 1. Consider the special case $\lambda=(1)$, which corresponds to the Schrödinger operator

$$
\begin{aligned}
\mathscr{L}_{(1)} & =-\frac{\mathrm{d}^{2}}{\mathrm{~d} z^{2}}-2 \frac{\mathrm{d}^{2}}{\mathrm{~d} z^{2}}\left(\log \left(2 z e^{-z^{2} / 2}\right)\right)+z^{2} \\
& =-\frac{\mathrm{d}^{2}}{\mathrm{~d} z^{2}}+z^{2}+\frac{2}{z^{2}}+2 .
\end{aligned}
$$

Already in this simple example, we obtain eigenfunctions (12) with a singularity on the real line (at $z=0$ ). Indeed, this can be seen explicitly by writing out the first exceptional Hermite polynomials $H_{(1), k}=\mathrm{Wr}\left(\psi_{k}, \psi_{1}\right)$ and the corresponding few eigenfunctions $\psi_{(1), k}=\frac{\operatorname{Wr}\left(\psi_{k}, \psi_{1}\right)}{\psi_{1}}$ :

$$
\begin{array}{ll}
H_{(1), 0}=1, & \psi_{(1), 0}=\frac{1}{z} e^{-z^{2} / 2}, \\
H_{(1), 2}=-\left(2+4 z^{2}\right), & \psi_{(1), 2}=-\frac{2+4 z^{2}}{z} e^{-z^{2} / 2}, \\
H_{(1), 3}=-16 z^{3}, & \psi_{(1), 3}=-16 z^{2} e^{-z^{2} / 2}, \\
H_{(1), 4}=12\left(1+4 z^{2}-4 z^{4}\right), & \psi_{(1), 4}=\frac{12\left(1+4 z^{2}-4 z^{4}\right)}{z} e^{-z^{2} / 2}, \\
H_{(1), 5}=64 z^{3}\left(5-2 z^{2}\right), & \psi_{(1), 5}=64 z^{2}\left(5-2 z^{2}\right) e^{-z^{2} / 2}, \\
H_{(1), 6}=-40\left(3+18 z^{2}-36 z^{4}+8 z^{6}\right), & \psi_{(1), 6}=-\frac{40\left(3+18 z^{2}-36 z^{4}+8 z^{6}\right)}{z} e^{-z^{2} / 2} .
\end{array}
$$

More generally, using the fact that

$$
\operatorname{Wr}\left(\psi_{2 l+1}, \psi_{1}\right)(-z)=-\operatorname{Wr}\left(\psi_{2 l+1}, \psi_{1}\right)(z), \quad l \in \mathbb{N},
$$

as well as the fact that each classical Hermite polynomial $H_{2 l}(z), l \in \mathbb{Z}_{\geq 0}$, has a non-zero constant term, it is readily seen that $\psi_{(1), l}(x)$ is regular on the whole real line if and only if $l \in \mathbb{Z}_{\geq 0} \backslash\{1\}$ is odd. The eigenvalues of the first few eigenfunctions are given in Figure 1, where open and filled circles indicate that the corresponding eigenfunctions are singular and non-singular, respectively. In addition, the cross represents the eigenvalue removed by the Darboux transformation. 


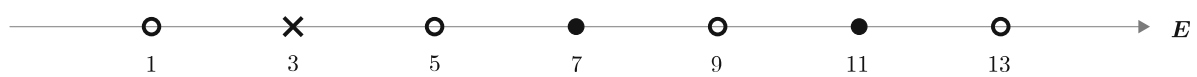

Figure 1. The eigenvalues of the first few eigenfunctions for $\lambda=(1)$.

Note that in the theory of quantum Calogero-Moser systems (of which this example is the simplest case) only non-singular solutions are considered (see e.g. [16]).

We will now use the fact that $\mathscr{D}_{\lambda}$ is obtained as the composition of first-order intertwining operators. To be more specific, let us introduce the short-hand notation

$$
\mathscr{W}_{m}=\operatorname{Wr}\left(\psi_{k_{m}}, \ldots, \psi_{k_{n}}\right), \quad \mathscr{W}_{m}(\psi)=\operatorname{Wr}\left(\psi, \psi_{k_{m}}, \ldots, \psi_{k_{n}}\right),
$$

(where it is convenient to allow $m=n+1$ and set $\mathscr{W}_{n+1}=1, \mathscr{W}_{n+1}(\psi)=\psi$ ), and recall the standard identity

$$
\mathscr{W}_{m-1} \mathscr{W}_{m}(\psi)=\mathscr{W}_{m-1}(\psi) \frac{\mathrm{d}}{\mathrm{d} x} \mathscr{W}_{m}-\mathscr{W}_{m} \frac{\mathrm{d}}{\mathrm{d} x} \mathscr{W}_{m-1}(\psi), \quad m \geq 1 .
$$

Then it is readily verified that

$$
\mathscr{D}_{\lambda}=D_{1} \circ \cdots \circ D_{m} \circ \cdots \circ D_{n},
$$

with

$$
D_{m}=\frac{\mathrm{d}}{\mathrm{d} z}-\frac{\mathrm{d}}{\mathrm{d} z}\left(\log \frac{\mathscr{W}_{m}}{\mathscr{W}_{m+1}}\right) .
$$

For our purposes, a key notion is that of trivial monodromy, see e.g. [19]. A Schrödinger operator $\mathscr{L}=-\mathrm{d}^{2} / \mathrm{d} z^{2}+u(z)$, whose potential $u$ is a meromorphic function of $z$, is said to have trivial monodromy if all solutions of its eigenvalue equation

$$
\mathscr{L} \psi(z)=E \psi(z)
$$

are meromorphic in $z$ for all $E$.

We recall that every monodromy-free Schrödinger operator $\mathscr{L}$ with a quadratically increasing rational potential is of the form (10) for some partition $\lambda$. The fact that each Schrödinger operator $\mathscr{L}_{\lambda}$ has trivial monodromy is easily seen. Indeed, in the special case $u(z)=z^{2}$ all eigenfunctions are entire, and trivial monodromy is preserved under (rational) Darboux transformations. The converse result is due to Oblomkov [15].

Duistermaat and Grünbaum [7] obtained local conditions for trivial monodromy. Specifically, in a neighbourhood of a pole $z=z_{i}$ the potential $u(z)$ must have a Laurent series expansion of the form

$$
u(z)=\sum_{r \geq-2} c_{r}\left(z-z_{i}\right)^{r},
$$


with

$$
c_{-2}=m_{i}\left(m_{i}+1\right) \text { for some } m_{i} \in \mathbb{N},
$$

and

$$
c_{2 j-1}=0, \quad \forall j=0,1, \ldots, m_{i} .
$$

In addition, every eigenfunction $\psi$ has a Laurent series expansion of the form

$$
\psi(z)=\left(z-z_{i}\right)^{-m_{i}} \sum_{r=0}^{\infty} \mathrm{d}_{r}\left(z-z_{i}\right)^{r}
$$

with

$$
\mathrm{d}_{2 j-1}=0, \quad \forall j=1, \ldots, m_{i} .
$$

We proceed to consider the implications for the CEHPs $H_{\lambda, l}$. Let $Z_{\lambda}$ be the set of zeros $z_{i} \in \mathbb{C}$ of the Wronskian $W_{\lambda}(z)$ with multiplicities $m_{i} \in \mathbb{N}$. In addition, we need the subset $Z_{\lambda}^{\mathbb{R}} \subset Z_{\lambda}$ obtained by restriction to $z_{i} \in \mathbb{R}$. We say that a meromorphic function $\psi(z)$ is quasi-invariant at the point $z=z_{i}$ with multiplicity $m_{i} \in \mathbb{N}$ if it satisfies the following two conditions:

(1) $\psi(z)\left(z-z_{i}\right)^{m_{i}}$ is analytic at $z=z_{i}$,

(2) $\left.\left(\psi(z)\left(z-z_{i}\right)^{m_{i}}\right)^{(2 j-1)}\right|_{z=z_{i}}=0$, for all $j=1, \ldots, m_{i}$.

The second condition can be rewritten as

$$
\psi\left(\sigma_{i}(z)\right)=(-1)^{m_{i}} \psi(z)+o\left(\left(z-z_{i}\right)^{m_{i}}\right),
$$

where $\sigma_{i}(z)=2 z_{i}-z$ is the reflection with respect to $z_{i}$. This explains the terminology.

Introducing the subspace

$$
\mathcal{Q}_{\lambda}=\left\{p \in \mathbb{C}[z]: \psi(z):=p(z) \frac{e^{-z^{2} / 2}}{W_{\lambda}(z)} \text { is quasi-invariant at } z=z_{i}, \quad \forall z_{i} \in Z_{\lambda}\right\},
$$

it follows from the above that the $\mathbb{C}$-linear span

$$
\mathcal{U}_{\lambda}=\left\langle H_{\lambda, l}: l \in \mathbb{Z}_{\geq 0} \backslash\left\{k_{1}, \ldots, k_{n}\right\}\right\rangle
$$

belongs to $\mathcal{Q}_{\lambda}$. From Proposition 5.3 in [12], we recall that the codimension of $\mathcal{U}_{\lambda}$ in $\mathbb{C}[z]$ is equal to $|\lambda|$. On the other hand, $|\lambda|$ is the degree of $W_{\lambda}(z)$, and therefore the number of quasi-invariance conditions that any $p \in \mathcal{Q}_{\lambda}$ should satisfy. This yields the converse inclusion, and thus the following result.

PROPOSITION 1. The $\mathbb{C}$-linear span of CEHPs coincides with polynomial quasiinvariants:

$$
\mathcal{U}_{\lambda}=\mathcal{Q}_{\lambda}
$$


Using the fact that the operator $T_{\lambda}$ [cf. (14)] preserves $\mathcal{U}_{\lambda}$, a different characterisation of this subspace was deduced in Proposition 5.4 in [12]: a polynomial $p \in \mathbb{C}[z]$ is an element of $\mathcal{U}_{\lambda}$ if only if

$$
2 W_{\lambda}^{\prime}\left(p^{\prime}-2 z p\right)-W_{\lambda}^{\prime \prime} p \in\left(W_{\lambda}\right),
$$

where $\left(W_{\lambda}\right)$ denotes the ideal generated by $W_{\lambda}$. That this divisibility condition is necessary is clear from the form of the singular part of $T_{\lambda}$ and that it is sufficient follows from a direct count of the number of linear conditions it imposes on a polynomial $p \in \mathbb{C}[z]$.

Whenever $\lambda$ is not a double partition, the Wronskian $W_{\lambda}(z)$ will have one or more real zeros [1], so that the weight function (4) is no longer non-singular on the real line. A formula for the precise number of real zeros was recently obtained in [13]. To resolve the problem with singularities, we replace the standard contour $\mathbb{R}$ by a shifted contour $C=i \xi+\mathbb{R}$ and consider a corresponding Hermitian product (5). As will become clear below, to ensure that the product is Hermitian we need to restrict attention to the following subspace of quasi-invariant polynomials:

$$
\mathcal{Q}_{\lambda, \mathbb{R}}=\left\{p \in \mathbb{C}[z]: \psi(z):=p(z) \frac{e^{-z^{2} / 2}}{W_{\lambda}(z)} \text { is quasi-invariant at } z=z_{i}, \quad \forall z_{i} \in Z_{\lambda}^{\mathbb{R}}\right\} .
$$

By counting quasi-invariance conditions, we obtain the next proposition.

PROPOSITION 2. The codimension of $\mathcal{Q}_{\lambda}$ in $\mathcal{Q}_{\lambda, \mathbb{R}}$ is $|\lambda|-\sum_{z_{i} \in Z_{\lambda}^{\mathbb{R}}} m_{i}$.

We are now ready for the main definition of this section.

DEFINITION 1. Let $\xi \in \mathbb{R}$ be such that

$$
0<|\xi|<\left|\operatorname{Im} z_{i}\right|, \quad \forall z_{i} \in Z_{\lambda} \backslash Z_{\lambda}^{\mathbb{R}} .
$$

Then, we define a sesquilinear product $\langle\cdot, \cdot\rangle$ on $\mathcal{Q}_{\lambda, \mathbb{R}}$ by setting

$$
\langle p, q\rangle=\int_{i \xi+\mathbb{R}} p(z) \bar{q}(z) \frac{e^{-z^{2}}}{W_{\lambda}^{2}(z)} \mathrm{d} z, \quad p, q \in \mathcal{Q}_{\lambda, \mathbb{R}} .
$$

Now we will show that the product does not depend on the specific choice of $\xi$. We find it worth stressing that this important property relies on our restriction to the subspace $\mathcal{Q}_{\lambda, \mathbb{R}}$.

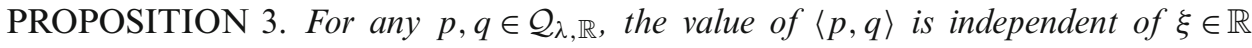
provided condition (18) is satisfied.

Proof. Let $I_{\xi}$ denote the integral in the right-hand side of (19). By Cauchy's theorem, it suffices to show that $I_{\xi}-I_{-\xi}=0$ for some $\xi$ satisfying (18). From the 
residue theorem, we deduce that the difference between the two integrals is proportional to

$$
\sum_{z_{i} \in Z_{\lambda, \mathbb{R}}} \operatorname{Res}_{z=z_{i}}\left(p(z) \bar{q}(z) \frac{e^{-z^{2}}}{W_{\lambda}^{2}(z)}\right) .
$$

We claim that each of these residues vanish. In fact, we have the following more general result.

LEMMA 1. If $\psi, \phi$ are quasi-invariant at $z=z_{i}$, with any multiplicity $m_{i} \in \mathbb{N}$, then

$$
\operatorname{Res}_{z=z_{i}}(\psi(z) \phi(z))=0 .
$$

Indeed, it follows from Condition (2) above that

$$
\begin{aligned}
& \left.\left(\psi(z) \phi(z)\left(z-z_{i}\right)^{2 m_{i}}\right)^{\left(2 m_{i}-1\right)}\right|_{z=z_{i}} \\
& \quad=\left.\left.\sum_{j=0}^{2 m_{i}-1}\left(\begin{array}{c}
2 m_{i}-1 \\
j
\end{array}\right)\left(\psi(z)\left(z-z_{i}\right)^{m_{i}}\right)^{\left(2 m_{i}-1-j\right)}\right|_{z=z_{i}}\left(\phi(z)\left(z-z_{i}\right)^{m_{i}}\right)^{(j)}\right|_{z=z_{i}} \\
& =0 .
\end{aligned}
$$

It is now straightforward to show that Definition 1 yields a Hermitian product.

PROPOSITION 4. The sesquilinear product $\langle\cdot, \cdot\rangle$ is Hermitian:

$$
\langle p, q\rangle=\overline{\langle q, p\rangle}, \quad \forall p, q \in \mathcal{Q}_{\lambda, \mathbb{R}} .
$$

Proof. In what follows, we find it convenient to use the notation

$$
w(z)=\frac{e^{-z^{2}}}{W_{\lambda}^{2}(z)},
$$

and use a subscript to indicate the choice of $\xi$ in (19). Since the classical Hermite polynomials have real coefficients, it is evident from (3) that $\bar{w}(z)=w(z)$. Hence, we have the following equalities:

$$
\begin{aligned}
\langle p, q\rangle_{\xi} & =\int_{\mathbb{R}} p(i \xi+x) \bar{q}(i \xi+x) w(i \xi+x) \mathrm{d} x \\
& =\int_{\mathbb{R}} \bar{p}(-i \xi+x) q(-i \xi+x) w(-i \xi+x) \mathrm{d} x \\
& =\overline{\langle q, p\rangle_{-\xi}} .
\end{aligned}
$$

Combined with Proposition 3, this yields the asserted hermiticity property. 
We recall that the classical Hermite polynomials $H_{l}(x)$ satisfy the orthogonality relation

$$
\int_{\mathbb{R}} H_{j}(x) H_{l}(x) e^{-x^{2}} \mathrm{~d} x=\delta_{j l} 2^{l} l ! \sqrt{\pi}, \quad j, l \in \mathbb{Z}_{\geq 0} .
$$

Combining this fact with the factorisation (15) of the intertwining operator $\mathscr{D}_{\lambda}$, it is now readily established by induction on the length $n$ of $\lambda$ that the CEHPs $H_{\lambda, l}(x)$ are orthogonal with respect to the Hermitian form $\langle\cdot, \cdot\rangle$ (cf. [12]).

THEOREM 1. The CEHPs $H_{\lambda, l}$ satisfy the orthogonality relation

$$
\left\langle H_{\lambda, j}, H_{\lambda, l}\right\rangle=\delta_{j l} \sqrt{\pi} 2^{l} l ! \prod_{m=1}^{n} 2\left(l-k_{m}\right), \quad j, l \in \mathbb{Z}_{\geq 0} \backslash\left\{k_{1}, \ldots, k_{n}\right\} .
$$

Proof. The assertion clearly holds true for $n=0$, with the empty product taken to be equal to one. Introducing the partition

$$
\widehat{\lambda}=\left(\lambda_{2}, \ldots, \lambda_{n}\right),
$$

we have

$$
\left\langle H_{\lambda, j}, H_{\lambda, l}\right\rangle=\int_{i \xi+\mathbb{R}}\left(D_{1} \psi_{\widehat{\lambda}, j}\right)(z)\left(\overline{D_{1} \psi_{\widehat{\lambda}, l}}\right)(z) \mathrm{d} z .
$$

Since $\overline{\mathscr{W}_{m}}=\mathscr{W}_{m}$, the (formal) adjoint of $D_{1}$ is given by

$$
D_{1}^{*}=-\frac{\mathrm{d}}{\mathrm{d} x}-\frac{\mathrm{d}}{\mathrm{d} x}\left(\log \frac{\mathscr{W}_{1}}{\mathscr{W}_{2}}\right) .
$$

The factorisation

$$
D_{1}^{*} D_{1}=\mathscr{L}_{\bar{\lambda}}-2 k_{1}-1
$$

thus entails that

$$
\left\langle H_{\lambda, j}, H_{\lambda, l}\right\rangle=2\left(l-k_{1}\right)\left\langle H_{\widehat{\lambda}, j}, H_{\widehat{\lambda}, l}\right\rangle .
$$

This completes the induction step, and the theorem is proved.

Remark 1. Since $\langle\cdot, \cdot\rangle$ is Hermitian, each squared norm $\langle p, p\rangle, p \in \mathcal{Q}_{\lambda, \mathbb{R}}$, is real, but need not to be positive. In fact, if the partition is not double, there is always a finite set of polynomials with negative squared norm, which can be easily identified using formula (21). For example, setting $\lambda=(1)$ in (21), we see that $\left\langle H_{(1), l}, H_{(1), l}\right\rangle<$ 0 if and only if $l=0$. Grinevich and Novikov [14] pointed out a similar fact in a finite-gap case. 
We conclude this section by showing that the linear $\operatorname{span} \mathcal{U}_{\lambda}$ is dense in $\mathcal{Q}_{\lambda, \mathbb{R}}$ in the sense that

$$
\left\langle p, H_{\lambda, l}\right\rangle=0, \quad \forall l \in \mathbb{Z}_{\geq 0} \backslash\left\{k_{1}, \ldots, k_{n}\right\} \Longrightarrow p \equiv 0 .
$$

By Proposition 1, we can formulate the result as follows.

THEOREM 2. The subspace $\mathcal{Q}_{\lambda}$ is dense in $\mathcal{Q}_{\lambda, \mathbb{R}}$.

Proof. Suppose that $p \in \mathcal{Q}_{\lambda, \mathbb{R}}$ is such that

$$
\langle p, q\rangle=0, \quad \forall q \in \mathcal{Q}_{\lambda} .
$$

Introducing the polynomials

$$
q_{\lambda, l}(z)=W_{\lambda}^{2}(z) H_{l}(z), \quad l \in \mathbb{Z}_{\geq 0},
$$

which clearly belong to the subspace $\mathcal{Q}_{\lambda}$, we obtain

$$
0=\left\langle p, q_{\lambda, l}\right\rangle=\int_{i \xi+\mathbb{R}} p(z) \bar{H}_{l}(z) e^{-z^{2}} \mathrm{~d} z, \quad \forall l \in \mathbb{Z}_{\geq 0} .
$$

Since the integrand is entire, we can take the limit $\xi \rightarrow 0$. Then expanding $p$ in terms of the classical Hermite polynomials $H_{l}$, it follows immediately from (20) that $p \equiv 0$.

Remark 2. If we assume that $\lambda$ is a double partition, then we recover orthogonality and completeness results from [12] (see Propositions 5.7, 5.8). Indeed, to recover the former it is enough to note that the weight function (4) is guaranteed to be non-singular on the real line, so that we can take the limit $\xi \rightarrow 0$ in (19); and the latter follows from the observation that we have $\mathcal{Q}_{\lambda, \mathbb{R}}=\mathbb{C}[z]$.

\section{Exceptional Laurent Orthogonal Polynomials}

In this section, we generalise our approach to the space of Laurent polynomials $\Lambda=\mathbb{C}\left[z, z^{-1}\right]$ using the trigonometric monodromy-free Schrödinger operators [4], which play an important role in the theory of Huygens' principle [2].

More specifically, we consider the Laurent polynomials $P_{\kappa, a ; l}$, as defined in (8). Due to the results of Theorem 3 and Proposition 6 we call $P_{\kappa, a ; l}, l \in \mathbb{Z}$, exceptional Laurent orthogonal polynomials (ELOPs).

\subsection{THE GENERAL CASE}

In this first subsection, we allow any choice of complex parameters $a=\left(a_{1}, \ldots, a_{n}\right)$, $a_{k} \in \mathbb{C} \backslash\{0\}$. 
We start from the elementary fact that the exponential functions

$$
e_{l}(x)=\exp (i l x), \quad l \in \mathbb{Z},
$$

have the eigenfunction property

$$
\mathscr{L} e_{l} \equiv-\frac{\mathrm{d}^{2} e_{l}}{\mathrm{~d} x^{2}}=l^{2} e_{l}, \quad x \in \mathbb{C} / 2 \pi \mathbb{Z} .
$$

Note that instead of usual unit circle $\mathbb{R} / 2 \pi \mathbb{Z}$ we consider its complex version cylinder $\mathbb{C} / 2 \pi \mathbb{Z}$. It is natural from the trivial monodromy point of view, see [4].

Sequences of Darboux transformations at the levels $0<k_{n}<k_{n-1}<\cdots<k_{1}$ are now parameterised by $n$ complex parameters $\theta=\left(\theta_{1}, \ldots, \theta_{n}\right), \theta_{k} \in \mathbb{C}$. Specifically, introducing the functions

$$
\phi_{k_{j}}\left(\theta_{j}, x\right)=2 \cos \left(k_{j} x+\theta_{j}\right), \quad j=1, \ldots, n,
$$

the resulting Schrödinger operator takes the form

$$
\mathscr{L}_{\kappa}=-\frac{\mathrm{d}^{2}}{\mathrm{~d} x^{2}}-2 \frac{\mathrm{d}^{2}}{\mathrm{~d} x^{2}}\left(\log \operatorname{Wr}\left(\phi_{k_{1}}, \ldots, \phi_{k_{n}}\right)\right),
$$

where $\kappa=\left\{k_{1}, \ldots, k_{n}\right\}$. Furthermore, letting $\mathscr{D}_{\kappa}$ act by

$$
\mathscr{D}_{\kappa} \phi=\frac{\operatorname{Wr}\left(\phi, \phi_{k_{1}}, \ldots, \phi_{k_{n}}\right)}{\operatorname{Wr}\left(\phi_{k_{1}}, \ldots, \phi_{k_{n}}\right)},
$$

the intertwining relation (11) holds true, and the functions

$$
\phi_{\kappa, \theta ; l}=\frac{\operatorname{Wr}\left(e_{l}, \phi_{k_{1}}, \ldots, \phi_{k_{n}}\right)}{\operatorname{Wr}\left(\phi_{k_{1}}, \ldots, \phi_{k_{n}}\right)}, \quad l \in \mathbb{Z},
$$

have the eigenfunction property

$$
\mathscr{L}_{\kappa} \phi_{\kappa, l}=l^{2} \phi_{\kappa, l} .
$$

We note that at each level $k_{j}, j=1, \ldots, n$, the multiplicity is reduced from two to one. Indeed, by (22)-(24) and linearity of the Wronskian, we have the relation

$$
\exp \left(i \theta_{j}\right) \phi_{\kappa, k_{j}}(x)+\exp \left(-i \theta_{j}\right) \phi_{\kappa,-k_{j}}(x) \equiv 0, \quad j=1, \ldots, n .
$$

To establish the precise connection between the functions $\phi_{\kappa, l}$ and the ELOPs $P_{\kappa, a ; l}$ given by (8), we change variable to

$$
z=\exp (i x)
$$

and fix the values of the parameters $a=\left(a_{1}, \ldots, a_{n}\right)$ according to

$$
a_{k}=\exp \left(i \theta_{k}\right) \in \mathbb{C} \backslash\{0\}, \quad k=1, \ldots, n .
$$


Then, it is readily seen that

$$
\phi_{\kappa, \theta ; l}(x)=P_{\kappa, a ; l}(z) \mathcal{W}_{\kappa, a}(z)^{-1},
$$

with $P_{\kappa, a ; l}(z)$ given by (8) and

$$
\mathcal{W}_{\kappa, a}(z)=\left|\begin{array}{cccc}
\Phi_{k_{1}}\left(a_{1} ; z\right) & \Phi_{k_{2}}\left(a_{2} ; z\right) & \cdots & \Phi_{k_{n}}\left(a_{n} ; z\right) \\
D \Phi_{k_{1}}\left(a_{1} ; z\right) & D \Phi_{k_{2}}\left(a_{2} ; z\right) & \cdots & D \Phi_{k_{n}}\left(a_{n} ; z\right) \\
\vdots & \vdots & \ddots & \vdots \\
D^{n-1} \Phi_{k_{1}}\left(a_{1} ; z\right) & D^{n-1} \Phi_{k_{2}}\left(a_{2} ; z\right) & \cdots & D^{n-1} \Phi_{k_{n}}\left(a_{n} ; z\right)
\end{array}\right|,
$$

where $D=z \mathrm{~d} / \mathrm{d} z$ and

$$
\Phi_{k}(a ; z)=a z^{k}+a^{-1} z^{-k}
$$

Furthermore, a direct computation reveals that $P_{\kappa, a ; l}$ is an eigenfunction of the operator

$$
T_{\kappa}=D^{2}-2 \frac{D \mathcal{W}_{\kappa, a}}{\mathcal{W}_{\kappa, a}} D+\frac{D^{2} \mathcal{W}_{\kappa, a}}{\mathcal{W}_{\kappa, a}}
$$

with eigenvalue $l^{2}$.

EXAMPLE 2. In the particular case $\kappa=\{1\}$ the corresponding Schrödinger operator is given by

$$
\begin{aligned}
\mathscr{L}_{\{1\}} & =-\frac{\mathrm{d}^{2}}{\mathrm{~d} x^{2}}-2 \frac{\mathrm{d}^{2}}{\mathrm{~d} x^{2}}\left(\log \left(2 \cos \left(x+\theta_{1}\right)\right)\right) \\
& =-\frac{\mathrm{d}^{2}}{\mathrm{~d} x^{2}}+\frac{2}{\cos ^{2}\left(x+\theta_{1}\right)} .
\end{aligned}
$$

When expressed in terms of the variable $z$ and the parameter $a_{1}$, the first few exceptional Laurent polynomials $P_{\{1\}, a ; l}$ defined by (8) and the corresponding eigenfunctions $\Phi_{\{1\}, a ; l}=P_{\{1\}, a ; l} / \Phi_{1}, l \in \mathbb{Z}$ are given by

$$
\begin{array}{ll}
P_{\{1\}, a ; 0}=a_{1} z-a_{1}^{-1} z^{-1}, & \Phi_{\{1\}, a ; 0}=\frac{a_{1} z-a_{1}^{-1} z^{-1}}{a_{1} z+a_{1}^{-1} z^{-1}}, \\
P_{\{1\}, a ;-1}=2 a_{1}, & \Phi_{\{1\}, a ;-1}=\frac{2 a_{1}}{a_{1} z+a_{1}^{-1} z^{-1}}, \\
P_{\{1\}, a ; 1}=-2 a_{1}^{-1}, & \Phi_{\{1\}, a ; 1}=-\frac{2 a_{1}^{-1}}{a_{1} z+a_{1}^{-1} z^{-1}}, \\
P_{\{1\}, a ;-2}=a_{1}^{-1} z^{-3}+3 a_{1} z^{-1}, & \Phi_{\{1\}, a ;-2}=\frac{a_{1}^{-1} z^{-3}+3 a_{1} z^{-1}}{a_{1} z+a_{1}^{-1} z^{-1}}, \\
P_{\{1\}, a ; 2}=a_{1} z^{3}+3 a_{1}^{-1} z, & \Phi_{\{1\}, a ; 2}=-\frac{a_{1} z^{3}+3 a_{1}^{-1} z}{a_{1} z+a_{1}^{-1} z^{-1}} .
\end{array}
$$

From these explicit formulae, it is evident that $P_{\{1\}, a ;-1}$ and $P_{\{1\}, a ; 1}$, as well as $\Phi_{\{1\}, a ;-1}$ and $\Phi_{\{1\}, a ; 1}$, are linearly dependent. Moreover, the definition of $\Phi_{\{1\}, a ; l}$ clearly entails that each such eigenfunction is singular at $z= \pm i / a_{1}$. 
We note that, upon setting

$$
\mathscr{W}_{m}=\operatorname{Wr}\left(\phi_{k_{m}}, \ldots, \phi_{k_{n}}\right),
$$

the intertwining operator $\mathscr{D}_{\kappa}$ factorises according to (15), (16). Just as in the Hermite case, it follows that each Schrödinger operator $\mathscr{L}_{\kappa}$ has trivial monodromy. Moreover, every monodromy-free trigonometric Schrödinger operator is of the form (23); see [4].

Let $Z_{\kappa}$ be the set of zeros $z_{i} \in \mathbb{C}$ of the function $\mathcal{W}_{\kappa, a}(z)$ with multiplicities $m_{i} \in$ $\mathbb{N}$ and $X_{\kappa}$ be the corresponding set consisting of $x_{j}$ such that $\exp \left(i x_{j}\right)=z_{j}, z_{j} \in$ $Z_{\kappa}$ (we drop the dependence on $a$ in the notations for brevity in the rest of this section).

Introduce the subspace

$$
\mathscr{Q}_{\kappa}=\left\{P \in \Lambda: \Phi(x):=\left(P / \mathcal{W}_{\kappa}\right)(\exp (i x)) \text { is quasi-invariant at all } x_{j} \in X_{\kappa}\right\} .
$$

It follows from the trivial monodromy property that

$$
\mathcal{U}_{\kappa}:=\left\langle P_{\kappa, l}: l \in \mathbb{Z}\right\rangle \subset \mathscr{Q}_{\kappa} .
$$

However, in contrast to Hermite case (see Proposition 1), the converse inclusion does not hold. Instead, we have the following result.

PROPOSITION 5. The codimension of $\mathcal{U}_{\kappa}$ in $\mathscr{Q}_{\kappa}$ is $n$.

Proof. From (8), we deduce that

$$
P_{\kappa, l}(z)=z^{l+|\kappa|} \operatorname{det} V\left(l, k_{1}, \ldots, k_{n}\right) \prod_{j=1}^{n} k_{j}+1 . \text { d. },
$$

where

$$
|\kappa|=\sum_{i=1}^{n} k_{i},
$$

1.d. stands for terms of lower degree and $V$ is the Vandermonde matrix

$$
V\left(\alpha_{1}, \ldots, \alpha_{m}\right)=\left[\begin{array}{cccc}
1 & 1 & \cdots & 1 \\
\alpha_{1} & \alpha_{2} & \cdots & \alpha_{m} \\
\vdots & \vdots & \ddots & \vdots \\
\alpha_{1}^{m-1} & \alpha_{2}^{m-1} & \cdots & \alpha_{m}^{m-1}
\end{array}\right] .
$$

Since $\operatorname{det} V\left(l, k_{1}, \ldots, k_{n}\right)=0$ if and only if $l=k_{1}, \ldots, k_{n}$, it follows that the degree sequence

$$
I_{\kappa}^{+}=\left\{\operatorname{deg} P(z): P \in \mathcal{U}_{\kappa}\right\}
$$


stabilises at $k_{1}+|\kappa|+1$ in the sense that $l \in I_{\kappa}^{+}$for all $l \geq k_{1}+|\kappa|+1$. Applying the same line of reasoning to the Laurent polynomials $P_{\kappa,-l}(1 / z)$, we find that the same statement holds true for

$$
I_{\kappa}^{-}=\left\{\operatorname{deg} P\left(z^{-1}\right): P \in \mathcal{U}_{\kappa}\right\} .
$$

Among the ELOPs $P_{\kappa, l}$ with $|l|<k_{1}+|\kappa|+1$, a maximal set of linearly independent Laurent polynomials is given by

$$
\begin{aligned}
l \in\left\{k_{1}, \ldots, k_{n}\right\} \cup\left\{0, \pm 1, \ldots, \pm\left(k_{n}-1\right)\right\} & \cup\left\{ \pm\left(k_{n}+1\right), \ldots, \pm\left(k_{n-1}-1\right)\right\} \\
\cup & \cup \cup\left\{ \pm\left(k_{2}+1\right), \ldots, \pm\left(k_{1}-1\right)\right\} .
\end{aligned}
$$

The cardinality of this index set equals

$$
n+2 k_{n}-1+2\left(k_{n-1}-k_{n}-1\right)+\cdots+2\left(k_{1}-k_{2}-1\right)=2 k_{1}-n+1 .
$$

Observing that

$$
2 k_{1}+2|\kappa|+1-\left(2 k_{1}-n+1\right)=2|\kappa|+n,
$$

we conclude that the codimension of $\mathcal{U}_{\kappa}$ in $\Lambda$ is $2|\kappa|+n$.

On the other hand, counting quasi-invariance conditions, we find that the codimension of $\mathscr{Q}_{\kappa}$ in $\Lambda$ equals $2|\kappa|$ and so the assertion follows.

Remark 3. In contrast to the case of usual polynomials there are several definitions of the degree of a Laurent polynomial, but none of them are convenient for our purposes. Let us define the $L$-degree $\operatorname{Ldeg} P$ of a Laurent polynomial $P=$ $\sum_{i=p}^{q} c_{i} z^{i}$ with $c_{p} \neq 0, c_{q} \neq 0$ as $q$ if $q>-p$, and $p$ if $q<-p$. If $q=-p$ the $L$ degree is not well-defined since it could be both $p$ and $q$. Under these assumptions

$$
\operatorname{Ldeg} P_{\kappa, l}=|\kappa|+l, l \in \mathbb{N} \backslash \kappa, \quad \operatorname{Ldeg} P_{\kappa, l}=-|\kappa|+l,-l \in \mathbb{N} \backslash \kappa,
$$

otherwise it is not well-defined. Note that the polynomials $P_{\kappa, k_{j}}$ and $P_{\kappa,-k_{j}}$ with undefined $L$-degrees are linearly dependent.

Next, we consider a particular complex bilinear form on $\mathscr{Q}_{\kappa}$, given by (6) with $W=\mathcal{W}_{\kappa}$, and establish corresponding Laurent orthogonality relations. A related Fourier theory for more general algebro-geometric operators was studied by Grinevich and Novikov in [14].

DEFINITION 2. Let $\mu \in \mathbb{R}_{>0}$ be such that

$$
\mu \neq\left|z_{i}\right|, \quad \forall z_{i} \in Z_{\kappa} .
$$

Then, we define a complex bilinear form $(\cdot, \cdot)$ on $\mathscr{Q}_{\kappa}$ by setting

$$
(P, Q)=\frac{1}{2 \pi i} \oint_{C_{\mu}} P(z) Q(z) \mathcal{W}_{\kappa}^{-2}(z) \frac{\mathrm{d} z}{z}, \quad P, Q \in \mathscr{Q}_{\kappa},
$$


where

$$
C_{\mu}=\{z \in \mathbb{C}:|z|=\mu\}
$$

Substituting $z=\exp (i x)$ and following the line of reasoning used in the proof of Lemma 3, we readily find that the product is well-defined in the sense that it does not depend on the choice of $\mu$. More precisely, we have the following lemma.

LEMMA 2. For any $P, Q \in \mathscr{Q}_{\kappa}$, the value of $(P, Q)$ is independent of $\mu \in \mathbb{R}_{>0}$ provided (26) is satisfied.

We are now ready to state and prove the first of the main results in this section, which may be viewed as a natural analog of Theorem 1 .

THEOREM 3. The ELOPS $P_{\kappa, l}$ satisfy the Laurent orthogonality relation

$$
\left(P_{\kappa, j}, P_{\kappa, l}\right)=\delta_{j+l, 0} \prod_{m=1}^{n}\left(l^{2}-k_{m}^{2}\right), \quad j, l \in \mathbb{Z} .
$$

Proof. Just as in the proof of Theorem 1, we note that the assertion holds true for $n=0$, and proceed by induction on the length $n$ of $\kappa$. Letting $\widehat{\kappa}=\left(k_{2}, \ldots, k_{n}\right)$, we have

$$
\left(P_{\kappa, j}, P_{\kappa, l}\right)=\frac{1}{2 \pi} \int_{0}^{2 \pi}\left(D_{1} \phi_{\widehat{\kappa}, j}\right)(x)\left(D_{1} \phi_{\widehat{\kappa}, l}\right)(x) \mathrm{d} x .
$$

Making use of the factorisation

$$
D_{1}^{*} D_{1}=\mathscr{L}_{\widehat{\kappa}}-k_{1}^{2}
$$

with

$$
D_{1}^{*}=-\frac{\mathrm{d}}{\mathrm{d} x}-\frac{\mathrm{d}}{\mathrm{d} x}\left(\log \frac{\mathscr{W}_{1}}{\mathscr{W}_{2}}\right)
$$

the (formal) adjoint of $D_{1}$, we deduce

$$
\left(P_{\kappa, j}, P_{\kappa, l}\right)=\left(l^{2}-k_{1}^{2}\right)\left(P_{\widehat{\kappa}, j}, P_{\widehat{\kappa}, l}\right),
$$

which completes the induction step.

Remark 4. Having started from an eigenvalue problem with doubly degenerate eigenvalues, we have that $\left(P_{\kappa, l}, P_{\kappa,-l}\right)=0$ for some of the ELOPs $P_{\kappa, l}$. More specifically, it is evident from the theorem that this is the case if and only if $l= \pm k_{m}$, $m=1, \ldots, n$. 
Expanding on the result of Proposition 5, we proceed to establish the precise relationship between $\mathcal{U}_{\kappa}$ and $\mathscr{Q}_{\kappa}$. We begin with a general definition.

Let $V$ be a vector space over $\mathbb{C}$. Then $V$ is called complex Euclidean space if it is equipped with a non-degenerate bilinear form $B: V \otimes V \rightarrow \mathbb{C}$.

DEFINITION 3. Let $W \subset V$ be a subspace of complex Euclidean space $V$. We say that $V$ is a minimal complex Euclidean extension of $W$ if

$$
\operatorname{dim}\left(\left.\operatorname{ker} B\right|_{W}\right)=\operatorname{codim}_{V} W .
$$

For any linear space $W$ and bilinear form $B$ with non-trivial kernel

$$
K:=\operatorname{ker} B
$$

it is readily verified that there is a unique (up to isomorphisms) minimal complex Euclidean extension $V \supset W$. Letting $K^{*}$ denote the dual space of $K$, it can be realised as follows:

$$
V=K \oplus K^{*} \oplus W / K
$$

with the extension of $B$ determined by

$$
\left(k_{1}+\hat{k}_{1}+w_{1}, k_{2}+\hat{k}_{2}+w_{2}\right) \mapsto \hat{k}_{2}\left(k_{1}\right)+\hat{k}_{1}\left(k_{2}\right)+B\left(w_{1}, w_{2}\right),
$$

where $k_{1}, k_{2} \in K, \hat{k}_{1}, \hat{k}_{2} \in K^{*}$ and $w_{1}, w_{2} \in W$. Moreover, for each basis $k_{1}, \ldots, k_{n} \in$ $K$, there is a unique basis $\hat{k}_{1}, \ldots, \hat{k}_{n} \in K^{*}$ such that $\left(k_{j}, \hat{k}_{l}\right)=\delta_{j l}$.

EXAMPLE 3. Suppose that $\left.B\right|_{W}=0$, so that each vector $w \in W$ is isotropic. Then we have

$$
V \cong W \oplus W^{*}
$$

with

$$
B\left(w_{1}+\hat{w}_{1}, w_{2}+\hat{w}_{2}\right)=\hat{w}_{2}\left(w_{1}\right)+\hat{w}_{1}\left(w_{2}\right), \quad w_{1}, w_{2} \in W, \quad \hat{w}_{1}, \hat{w}_{2} \in W^{*} .
$$

As demonstrated by the following proposition, the inclusion $\mathcal{U}_{\kappa} \subset \mathscr{Q}_{\kappa}$ provides a concrete example of a minimal complex Euclidean extension in the sense of Definition 3 .

PROPOSITION 6. $\mathscr{Q}_{\kappa}$ is the minimal complex Euclidean extension of $\mathcal{U}_{\kappa}$.

Proof. From Theorem 3 we infer that

$$
\operatorname{ker}(\cdot, \cdot) \mid \mathcal{U}_{\kappa}=\left\langle P_{\kappa, k_{j}}: j=1, \ldots, n\right\rangle
$$


(Note the linear relations $a_{j} P_{\kappa, k_{j}}+a_{j}^{-1} P_{\kappa,-k_{j}}=0$.) Since ELOPs $P_{\kappa, l}$ corresponding to different values of $l^{2}$, and hence different eigenvalues, are linearly independent, it follows that

$$
\operatorname{dim}\left(\operatorname{ker}(\cdot, \cdot) \mid \mathcal{u}_{\kappa}\right)=n
$$

Recalling Proposition 5, we see that it remains only to verify that $(\cdot, \cdot)$ is nondegenerate on $\mathscr{Q}_{\kappa}$. Observing that

$$
\mathcal{W}_{\kappa}^{2}(z) z^{j} \in \mathscr{Q}_{\kappa}, \quad \forall j \in \mathbb{Z},
$$

we have

$$
\left(P, \mathcal{W}_{\kappa}^{2}(z) z^{-l}\right)=\frac{1}{2 \pi i} \oint_{C_{\mu}} P(z) z^{-l} \frac{d z}{z}
$$

which clearly is non-zero for all non-zero $P \in \mathscr{Q}_{\kappa}$, and suitable $l \in \mathbb{Z}$.

\subsection{THE HERMITIAN CASE}

In the case when all $\theta_{k}$ are real or, equivalently, when parameters $a=\left(a_{1}, \ldots, a_{n}\right)$ satisfy

$$
\left|a_{k}\right|=1, \quad k=1, \ldots, n,
$$

we can introduce the Hermitian structure as follows.

Note that in this case the weight function $w(z)=\mathcal{W}_{\kappa}(z)^{-2}$ is invariant under the antilinear involution

$$
P^{\dagger}(z):=\overline{P(1 / \bar{z})}, \quad P \in \Lambda,
$$

which will play much the same role as the Schwartz conjugate did in the Hermite case. In fact, observing that $(D P)^{\dagger}=-D P^{\dagger}$ and that $\Phi_{k}^{\dagger}=\Phi_{k}$, we can deduce from (25) that

$$
\mathcal{W}_{\kappa}^{\dagger}(z)=(-1)^{n(n-1) / 2} \mathcal{W}_{\kappa}(z), \quad \kappa=\left\{k_{1}, \ldots, k_{n}\right\} .
$$

In addition, the zero set $Z_{\kappa}$ becomes invariant under the involution $z \rightarrow 1 / \bar{z}$, i.e.

$$
z_{i} \in Z_{\kappa} \Longrightarrow 1 / \bar{z}_{i} \in Z_{\kappa},
$$

and, since $z=1 / \bar{z}$ whenever $|z|=1$, we have that

$$
\mathcal{W}_{\kappa}(z) \mathcal{W}_{\kappa}^{\dagger}(z)=\left|\mathcal{W}_{\kappa}\right|^{2}, \quad|z|=1 .
$$


Letting $Z_{\kappa}^{C}=\left\{z_{i} \in Z_{\kappa}:|z|=1\right\}$ and $X_{\kappa}^{\mathbb{R}}=\left\{x_{j}: \exp \left(i x_{j}\right)=z_{j}, z_{j} \in Z_{\kappa}^{C}\right\} \subset \mathbb{R}$, we introduce the following subspace of quasi-invariant Laurent polynomials:

$$
\mathscr{Q}_{\kappa, C}=\left\{P \in \Lambda: \Phi(x):=\left(P / \mathcal{W}_{\kappa}\right)(\exp (i x)) \text { is quasi-invariant at all } x_{j} \in X_{\kappa}^{\mathbb{R}}\right\} .
$$

From (32), it is straightforward to infer that

$$
\mathscr{Q}_{\kappa}^{\dagger}=\mathscr{Q}_{\kappa}, \quad \mathscr{Q}_{\kappa, C}^{\dagger}=\mathscr{Q}_{\kappa, C},
$$

which allows us to define a natural sesquilinear product on $\mathscr{Q}_{\kappa, C}$.

DEFINITION 4. Assuming that (30) holds true, we introduce

$$
v=\min _{\substack{z_{i} \in Z_{\kappa} \\\left|z_{i}\right|>1}}\left|z_{i}\right|
$$

and let $\mu \in \mathbb{R}_{>0}$ be such that

$$
1<\max (\mu, 1 / \mu)<v
$$

Then, we define a sesquilinear product $\langle\cdot, \cdot\rangle_{L}$ on $\mathscr{Q}_{\kappa, C}$ by setting

$$
\langle P, Q\rangle_{L}=\frac{1}{2 \pi i} \oint_{C_{\mu}} P(z) Q^{\dagger}(z)\left(\mathcal{W}_{\kappa}(z) \mathcal{W}_{\kappa}^{\dagger}(z)\right)^{-1} \frac{\mathrm{d} z}{z}, \quad P, Q \in \mathscr{Q}_{\kappa, C} .
$$

Again, the product does not depend on the specific choice of $\mu$.

LEMMA 3. For any $P, Q \in \mathscr{Q}_{\kappa, C}$, the value of $\langle P, Q\rangle_{L}$ is independent of $\mu \in \mathbb{R}_{>0}$ provided (34) is satisfied.

By adapting the proof of Proposition 4, we can use the lemma to show that Definition 4 yields a Hermitian product.

PROPOSITION 7. The sesquilinear product $\langle\cdot, \cdot\rangle_{L}$ is Hermitian:

$$
\langle P, Q\rangle_{L}=\overline{\langle Q, P\rangle_{L}}, \quad \forall P, Q \in \mathscr{Q}_{\kappa, C} .
$$

Proof. Using the notation

$$
w(z)=1 / \mathcal{W}_{\kappa}(z) \mathcal{W}_{\kappa}^{\dagger}(z)
$$


and using a subscript to indicate the choice of $\mu$ in (35), we deduce the following equalities:

$$
\begin{aligned}
\langle P, Q\rangle_{L, \mu} & =\frac{1}{2 \pi} \int_{0}^{2 \pi} P\left(\mu e^{i \varphi}\right) \bar{Q}\left(\mu^{-1} e^{-i \varphi}\right) w\left(\mu e^{i \varphi}\right) \mathrm{d} \varphi \\
& =\frac{1}{2 \pi} \int_{0}^{2 \pi} \bar{P}\left(\mu e^{-i \varphi}\right) Q\left(\mu^{-1} e^{i \varphi}\right) w\left(\mu^{-1} e^{i \varphi}\right) \mathrm{d} \varphi \\
& =\overline{\langle Q, P\rangle_{L, \mu^{-1}}},
\end{aligned}
$$

and so hermiticity follows from Lemma 3.

Moreover, the proof of Theorem 3 is readily adapted to yield the following orthogonality result.

THEOREM 4. Assuming that (30) holds true, the ELOPs $P_{\kappa, l}$ satisfy the orthogonality relation

$$
\left\langle P_{\kappa, j}, P_{\kappa, l}\right\rangle_{L}=\delta_{j l} \prod_{m=1}^{n}\left(k_{m}^{2}-l^{2}\right), \quad j, l \in \mathbb{Z} .
$$

Proof. Taking $z=\exp (i x)$ in the integral in (35) and observing that (cf. (32))

$$
\overline{\mathscr{W}_{m}}(-x)=(-1)^{(n-m)(n-m+1) / 2} \mathscr{W}_{m}(x),
$$

we establish the equalities

$$
\begin{aligned}
\left\langle P_{\kappa, j}, P_{\kappa, l}\right\rangle_{L} & =\frac{1}{2 \pi} \int_{0}^{2 \pi}\left(D_{1} \phi_{\widehat{\kappa}, j}\right)(x) \overline{\left(D_{1} \phi_{\widehat{\kappa}, l}\right)}(-x) \mathrm{d} x \\
& =-\frac{1}{2 \pi} \int_{0}^{2 \pi} \phi_{\widehat{\kappa}, j}(x) \overline{\left(D_{1}^{*} D_{1} \phi_{\widehat{\kappa}, l}\right)}(-x) \mathrm{d} x .
\end{aligned}
$$

Appealing to the factorisation (29), we thus obtain the relation

$$
\left\langle P_{\kappa, j}, P_{\kappa, l}\right\rangle_{L}=\left(k_{1}^{2}-l^{2}\right)\left\langle P_{\widehat{\kappa}, j}, P_{\widehat{\kappa}, l}\right\rangle_{L},
$$

and the assertion follows by induction on $n$.

After replacing the bilinear form $B$ by a Hermitian sesquilinear form $h$, Definition 3 as well as the succeeding discussion applies with minor changes also in the present situation. Specifically, we say that $V$ is a minimal Hermitian extension of $W$ if

$$
\operatorname{dim}\left(\left.\operatorname{ker} h\right|_{W}\right)=\operatorname{codim}_{V} W .
$$

Then, we have the following analog of Theorem 2 . 
THEOREM 5. The subspace $\mathscr{Q}_{\kappa}$, which is the minimal Hermitian extension of $\mathcal{U}_{\kappa}$, is dense in $\mathscr{Q}_{\kappa, C}$.

Proof. Suppose that $P \in \mathscr{Q}_{\kappa, C}$ is such that

$$
\langle P, Q\rangle_{L}=0, \quad \forall Q \in \mathscr{Q}_{\kappa} .
$$

Since the Laurent polynomials

$$
Q_{\kappa, l}=\mathcal{W}_{\kappa}(z) \mathcal{W}_{\kappa}^{\dagger}(z) z^{l}, \quad l \in \mathbb{Z},
$$

clearly are contained in $\mathscr{Q}_{\kappa}$, we have that

$$
0=\left\langle P, Q_{\kappa, l}\right\rangle_{L}=\frac{1}{2 \pi i} \oint_{C_{\mu}} P(z) z^{l} \frac{\mathrm{d} z}{z}, \quad \forall l \in \mathbb{Z} .
$$

Taking the limit $\mu \rightarrow 1$ and using the property that

$$
\frac{1}{2 \pi i} \oint_{|z|=1} z^{k} z^{l} \frac{\mathrm{d} z}{z}=\delta_{k+l, 0}, \quad k, l \in \mathbb{Z}
$$

we conclude that $P \equiv 0$.

Remark 5. It is known from the soliton theory that for every non-empty set $\kappa$ and any choice of real $\theta_{k}$ the corresponding potential always has singularities on the real line. This means that in the Laurent case we do not have non-trivial regular examples (unlike Hermite case with double partitions).

\section{Concluding Remarks}

We have discussed two complex versions of the exceptional orthogonal polynomials, related to two classes of monodromy-free Schrödinger operators. We would like to emphasise two novelties compared to the original approach of Gómez-Ullate et al. $[11,12]$.

First, in order to define the inner product in general we have to reduce the space of polynomials to the subspace of quasi-invariants, which has a finite codimension. The only exception is the Hermite case with double partitions considered in [12].

Second, in the Laurent case the space of quasi-invariants is not generated by the corresponding exceptional Laurent polynomials, so we need to consider the minimal complex Euclidean extension.

In the rational case with sextic growth at infinity there are some partial results [10], which lead to finite sets of orthogonal polynomials of the same degree. It would be interesting to analyse this situation in view of a recent very interesting paper by Felder and Willwacher [9]. 
It would be interesting also to see what happens with exceptional orthogonal polynomials in the multidimensional case. One can use the monodromy-free generalised Calogero-Moser operators, playing an important role in the theory of Huygens principle [4]. We plan to address this elsewhere soon.

\section{Acknowledgements}

APV is grateful to Oleg Chalykh and Leon Takhtajan for very useful and encouraging discussions. The work of $\mathrm{MH}$ and APV was partially supported by the EPSRC grants EP/K010123/1 and EP/J00488X/1, respectively.

Open Access This article is distributed under the terms of the Creative Commons Attribution License which permits any use, distribution, and reproduction in any medium, provided the original author(s) and the source are credited.

\section{References}

1. Adler, V.E.: A modification of Crum's method. Theor. Math. Phys. 101, 13811386 (1995)

2. Berest, Y.Y., Lutsenko, I.M.: Huygens' principle in Minkowski spaces and soliton solutions of the Korteweg-de Vries equation. Commun. Math. Phys. 190, 113-132 (1997)

3. Bochner, S.: Über Sturm-Liouvillesche Polynomsysteme. Math. Z. 29, 730-736 (1929)

4. Chalykh, O.A., Feigin, M.V., Veselov, A.P.: Multidimensional Baker-Akhiezer functions and Huygens' principle. Commun. Math. Phys. 206, 533-566 (1999)

5. Crum, M.M.: Associated Sturm-Liouville systems. Q. J. Math. Oxford Ser. 6, 121127 (1955)

6. Cruz-Barroso, R., Diaz Mendoza, C., Orive, R.: Orthogonal Laurent polynomials. A new algebraic approach. J. Math. Anal. Appl. 408, 40-54 (2013)

7. Duistermaat, J.J., Grünbaum, F.A.: Differential equations in the spectral parameter. Commun. Math. Phys. 103, 177-240 (1986)

8. Felder, G., Hemery, A.D., Veselov, A.P.: Zeros of Wronskians of Hermite polynomials and Young diagrams. Phys. D 241, 2131-2137 (2012)

9. Felder, G., Willwacher, T.: Jointly orthogonal polynomials. J. Lond. Math. Soc. 91(3), 750-768 (2015)

10. Gibbons, J., Veselov, A.P.: On the rational monodromy-free potentials with sextic growth. J. Math. Phys. 50(1), 013513 (2009)

11. Gómez-Ullate, D., Kamran, N., Milson, R.: An extension of Bochner's problem: exceptional invariant subspaces. J. Approx. Theory 162, 987-1006 (2010)

12. Gómez-Ullate, D., Grandati, Y., Milson, R.: Rational extensions of the quantum harmonic oscillator and exceptional Hermite polynomials. J. Phys. A 47, 015203 (2014)

13. García-Ferrero, M.Á., Gómez-Ullate, D.: Oscillation theorems for the Wronskian of an arbitrary sequence of eigenfunctions of Schrödinger's equation. Lett. Math. Phys. 105, 551-573 (2015)

14. Grinevich, P.G., Novikov, S.P.: Singular soliton operators and indefinite metrics. Bull. Braz. Math. Soc. N. Ser. 44(4), 809-840 (2013)

15. Oblomkov, A.A.: Monodromy-free Schrödinger operators with quadratically increasing potential. Theor. Math. Phys. 121, 1574-1584 (1999) 
16. Olshanetsky, M.A., Perelomov, A.M.: Quantum integrable systems related to Lie algebras. Phys. Rep. 94, 313-404 (1983)

17. Reed, M., Simon, B.: Methods of modern mathematical physics. I. Functional analysis, 2nd edn. Academic Press, New York (1980)

18. Szegö, G.: Orthogonal polynomials. AMS Colloquium Publ., AMS (1939)

19. Veselov, A.P.: On Stieltjes relations, Painlevé-IV hierarchy and complex monodromy. J. Phys. A 34, 3511-3519 (2001) 